\title{
Translating Research into Practice: The Colorectal Cancer Screening Experience
}

\author{
Navkiran K. Shokar, MA, MD, MPH \\ Paul L Foster School of Medicine, Texas Tech University Health Sciences Center, El Paso, TX, USA.
}

J Gen Intern Med 29(1):10-2

DOI: $10.1007 / \mathrm{s} 11606-013-2592-0$

(C) Society of General Internal Medicine 2013

\begin{abstract}
A lthough funding for research has increased dramatically over the last 30 years, the rate of its application to human health at the clinical or population level has lagged far behind. There are numerous examples of delays in the transfer of research-proven interventions to widespread application in routine clinical practice. ${ }^{1}$ This phenomenon has been labeled the "research valley of death," and the need to better understand and mitigate this delay in the process of "bench to bedside" applications has led to conceptualization of the translational research paradigm. This process has been described as consisting of a number of steps: the $\mathrm{T} 1$ to $\mathrm{T} 2$ step relates to the transfer of basic sciences discoveries to patients, and the $\mathrm{T} 2$ to $\mathrm{T} 3$ step represents the transfer of clinical research-proven interventions to widespread clinical practice. In this issue of JGIM, Hendren et al. ${ }^{3}$ describe the findings of a multimodal breast and colorectal cancer screening intervention conducted in an inner city primary care practice. They report an increase in screening rates of a statistically significant 21 percentage points for colorectal cancer screening and 13 percentage points for mammography (a non-significant increase). This study, the topic, and the health intervention highlight some of the challenges and issues associated with translating research into clinical practice.

Colorectal cancer (CRC) screening has been recommended since the 1990s; with cervical cancer screening, it is one of only two cancer screening recommendations that receive the highest grade of recommendation (category A) from the United States Preventive Services Task Force. ${ }^{4}$ Yet, despite 20 years of recommendations, CRC screening rates remain suboptimal, with the latest national data from the National Health Interview Survey (NHIS) revealing a CRC screening rate of less than $60 \%$, only a modest increase over the $41 \%$ rate seen in $2000 .{ }^{5,6}$ Screening rates are even lower in some groups: among
\end{abstract}

Published online August 24, 2013 recent immigrants, individuals without health insurance, and those lacking a regular source of care, screening rates fall below $25 \%$, and rates in minority populations remain lower than those in non-Hispanic whites. ${ }^{5}$

Although research demonstrating the benefit of screening interventions such as education, reminder letters, and mailed fecal occult blood test (FOBT) kits has existed since the $1990 \mathrm{~s}^{7}$, widespread uptake of these practices has not occurred. Unfortunately, as discussed above, this delay between proven clinical research and its uptake into routine clinical practice is not an isolated problem; this is a widespread phenomenon that has been described for all types of clinical conditions. ${ }^{1}$ The need to facilitate the T2 to T3 transfer of research to clinical practice has spurred the development of a relatively new field of research, termed implementation and dissemination science. This field seeks to understand the barriers and facilitators to the uptake of clinical research, and to develop new methods to facilitate the transfer.

Although there are a myriad of factors that account for the "research to clinical practice" delay, a factor central to the problem is the struggle between the need to establish efficacy (does an intervention work under ideal conditions) versus effectiveness (does it work in the real world). In order to transfer research knowledge to practice, wellconducted effectiveness studies are needed. Sackett and others have proposed the concept of pragmatic trials to test effectiveness. ${ }^{8}$ These trials differ from those conducted under the highly controlled or artificial conditions of an explanatory randomized controlled trial. They attempt to demonstrate what happens under usual conditions, so that clinicians and policymakers can be more confident that the findings are applicable and relevant to their own environment, and are therefore more likely to adopt them. An international group of trialists have developed a set of Pragmatic-Explanatory Continuum Indicators (PRECIS) ${ }^{9}$ to aid researchers in maximizing their research designs to address their goals. It is highly unlikely that a trial will be 
wholly pragmatic or explanatory; the reality is that most will fall somewhere in between. Ten domains are postulated to be important in designing a pragmatic versus an explanatory trial. These include: the eligibility criteria (least restrictive in a pragmatic trial), degree of flexibility in how the intervention is conducted (flexible in a pragmatic trial), degree to which practitioners are usual providers with usual expertise, degree to which the comparison intervention is usual care applied by usual practitioners, degree of followup intensity (the most pragmatic rely on administrative databases or chart review), degree to which the primary trial outcomes are objective and clinically meaningful, degree of unobtrusive compliance measurement without enhanced strategies to improve follow-up, degree of measurement of practitioner adherence or unobtrusive measurement without strategies to improve it, and intention to treat analysis. In terms of application, pragmatic trials are particularly important for settings where the population is relatively diverse in age, background, number of comorbidities - in other words, a situation that is commonplace in primary care and community settings.

Hendren et al. are to be congratulated for incorporating many of these components into the design of their study. They extracted components known to be efficacious under ideal conditions (reminder letters, automated phone calls, mailed FOBT kits, and physician and patient prompts) for application to their own real world setting. Their findings are generalizable to urban primary care practices throughout the US that take care of predominantly low income patients, because the findings are relevant and applicable, based on the inclusion of all clinically eligible participants, recruitment of usual practitioners, inclusion of a usual care comparison group, follow-up using chart review, clinically meaningful primary outcomes, an intention to treat analysis, and lack of enhanced practitioner based strategies beyond the intervention itself.

Pragmatic as this study was, however, additional information will be needed to assist practices in deciding whether it is feasible to apply these findings to their own settings. For instance, in this study the research team remained responsible for most of the activities, e.g. abstracting data to determine who was eligible, mailing letters of information to the patient, tracking patients to see if they completed testing, mailing the FOBT kits, developing the registry database, preparing the automated phone calls, and arranging the point of care prompts. In contrast to other types of clinical interventions (e.g., medication selection), organizational change interventions require a complex series of changes at the practice level. As the authors point out, organizational change has the greatest impact on preventive health improvements in practice as compared to approaches such as provider or patient reminders, education or incentives and provider feedback. ${ }^{10}$ However, in order for organizational change interventions to be adopted widely in clinical practice, it is very important that effectiveness studies provide clear information about the resources that were utilized, the infrastructure needed, the degree to which components were implemented as planned, the relative contribution of different components to screening, and the incremental costs in dollars. Addressing these issues in the future will become even more important as the number of comparative effectiveness studies increases over the next few years, under the auspices of the Patient Centered Outcomes Research Institute (PCORI). ${ }^{11}$

Organizational change certainly is the topic of the moment in primary care, with ongoing patient-centered medical home initiatives that include practice re-design activities such as team-based care, and integration of pharmacists, health educators, navigators, practice facilitators, and care managers into the care of the patients. ${ }^{12-14}$ This model, with its emphasis on population management, may make organizational interventions more feasible in the future, particularly as disease registry systems are adopted and population management personnel become an integral part of patient care teams.

Corresponding Author: Navkiran K. Shokar, MA, MD, MPH; Paul L Foster School of Medicine, Texas Tech University Health Sciences Center, 9849 Kenworthy Street, El Paso, TX 79924, USA (e-mail: navkiran.shokar@ttuhsc.edu).

\section{REFERENCES}

1. Avorn J, Fischer M. 'Bench to behavior': translating comparative effectiveness research into improved clinical practice. Health Aff (Millwood). 2010;29(10):1891-1900.

2. Butler D. Translational research: crossing the valley of death. Nature. 2008;453(7197):840-842.

3. Hendren S, Winters $\mathbf{P}$, Humiston S, et al. Randomized, Controlled Trial of a Multimodal Intervention to Improve Cancer Screening Rates in a Safety-Net Primary Care Practice. J Gen Intern Med. doi:10.1007/ s11606-013-2506-1.

4. Whitlock EP, Lin JS, Liles E, Beil TL, Fu R. Screening for colorectal cancer: a targeted, updated systematic review for the U.S. Preventive Services Task Force. Ann Intern Med. 2008;149(9):638-658.

5. Klabunde $\mathbf{C}$, Brown $\mathbf{M}$, Ballard-Barbash $\mathbf{R}$, et al. Cancer Screening-United States, 2010. Morb Mortal Wkly Rep. 2012;61(3):41-45.

6. Swan J, Breen N, Coates R, Rimer B, Lee N. Progress in cancer screening practices in the United States: Results from the 2000 National Health Interview Survey. Cancer. 2003;97:1528-1540.

7. Vernon S. Participation in colorectal cancer screening: a review. J Natl Cancer Inst. 1997;89:1406-1421.

8. Sackett D. Explanatory versus Management Trials. In: Haynes R, Sackett D, Guyatt G, Tugwell P, eds. Clinical Epidemiology: How to do Clinical Practice Research. Vol 424: Lippincott Williams \& Wilkins; 2006.

9. Thorpe $\mathbf{K}, \mathbf{Z}$ warenstein $\mathbf{M}$, Oxman A, et al. A pragmatic-explanatory continuum indicator summary (PRECIS): a tool to help trial designers. J Clin Epidemiol. 2008;62:464-475.

10. Stone EG, Morton SC, Hulscher ME, et al. Interventions That Increase Use of Adult Immunization and Cancer Screening Services: A MetaAnalysis. Ann Intern Med. 2002;136:641-651. 
11. PCORI. Patient Centered Outcomes Research Institute (PCORI). 2013. Accessed 7/26, 2013.

12. Taylor EF, Machta RM, Meyers DS, Genevro J, Peikes DN. Enhancing the Primary Care Team to Provide Redesigned Care: The Roles of Practice Facilitators and Care Managers. Ann Fam Med. 2013;11(1):80-83.
13. Rosland AM, Nelson $\mathbf{K}$, Sun $\mathbf{H}$, et al. The Patient-Centered Medical Home in the Veterans Health Administration. Am $\mathrm{J}$ Manag Care. 2013;19(7):e263-e272.

14. Davis K, Schoenbaum SC, Audet AM. A 2020 vision of patient-centered primary care. J Gen Intern Med. 2005;20(10):953-957. 